\title{
The Impact of Physical Parameters on the Perception of the Moving Elements in Peripheral Part of the Screen
}

\author{
Snježana IVANČIĆ VALENKO, Vladimir CVILJUŠAC, Sanja ZLATIĆ, Damir MODRIĆ
}

\begin{abstract}
Studying the importance of the color and shape perception with peripheral vision on the screen has an important role in digital design to provide designers with guidelines for better performance that will contribute to more functional user interfaces. In this paper, we have explored how user experience is affected by moving elements in the peripheral part of the screen, in correlation with other physical parameters that describe their movement. It is known that the motion of the object greatly increases its perception. This paper examines the influence of color, complexity of shape, speed and motion direction of shapes and other parameters on the perception of moving objects by peripheral vision. The attention of the examinees was focused on the central part of the screen by doing three types of tests. During the test, in the area of peripheral vision, moving elements were randomly generated which influenced the attentions of the examinees while testing the response rate and the quality of their perception. The expected preliminary results of peripheral perception studies confirm that numerous parameters such as color, position, shape, and speed of graphic elements affect their peripheral perception.
\end{abstract}

Keywords: perception; peripheral vision; user interface

\section{INTRODUCTION}

In this paper we will present a new method of the standardization of the correct answers in a test that studies the speed and accuracy of perception and reaction with peripheral vision. To get an objective value of correct answers, regardless of their number, we introduced a new method of answer number standardization using weighting factors that will be explained below.

Peripheral vision is the part of vision that is outside the center of gaze, i.e., point of fixation, and it is the largest part of the visual field. The normal visual field is about 170 degrees around, with 100 degrees of peripheral vision. Visual perception of objects in the peripheral space is poorer than the one in the central part. However, it has a significant impact on users, and high attention is given to appearance and layout of these objects in the visual space. When viewing and reading both central and peripheral vision are used.

There are significant differences in searching for objects in the central and peripheral part. It is easier to observe objects in the central part due to the greater sharpness of the vision, but also because of the fact that attention is naturally focused on this part [1].

This paper studies the influence of parameters such as color, complexity of shape and characteristics of their motion (listed in Tab. 1) that affect the level and quality of perception of moving elements in the peripheral part of the screen. The experiment was conducted in such a way that the attention of the respondents was focused on the center of the screen, while in the peripheral part, elements attracting the attention of the examinees randomly appeared. Furthermore, the obtained results show that the speed and accuracy of the reaction are influenced by numerous parameters, among which the complexity of the object is most significant.

Gutwin, Cockburn and Coveney [2] studied the identification accuracy of objects at different distances from the center of the screen (different eccentricities). The objects that appeared on different parts of the screen during the experiment varied in six parameters (hue, shape, size, luminance, flashing, motion). Experiments showed that in the middle of the screen almost all stimuli had a considerable identification accuracy. However, in the peripheral part, the motion, flashing, and luminance were much more noticeable than the color and shape. This shows how moving forms are perceived with greater accuracy; however, moving text is harder to read than static text.

Similar results were obtained by Levi, Klein, and Aitsebaomo. At the maximum distance from the point of fixation, the movement is the most noticeable, much more than other elements such as color and text [3].

Hansen, Pracejus and Gegenfurtner [4] point out that color perception is possible even at eccentricities up to 50 degrees along the L-M axis, but not as good as in the central part. In their experiment, the participants were focused on the white dot in the center of the screen, and in the peripheral part colored circles randomly appeared and had to be detected by pressing a predefined key of different sizes.

A study from 1977 conducted by Gordon and Abramov [5] shows that color vision in the peripheral part mostly depends on the size of the stimulus. If the stimulus is bigger, it will be perceived earlier. As the size of the stimulus decreases and the stimulus moves from the center to the periphery, green is the first color (hue) that cannot be identified, followed by yellow.

In his dissertation, Frey, N. W. [6] studied the visibility of colors in the area of peripheral vision, where all the colors used in the research (red, green, yellow or blue) appear on the outer edges of the periphery. The stimulus of a certain color is displayed at every $10^{\circ}$ of the screen, started from the farthest point on the screen toward the center. At each occurrence, the stimuli were visible for three seconds. The stimulus was always of the same color, but at the far points of focus it appeared gray, and as it approached the center point, it seemed to change the color tone. So for example, the green stimulus on the periphery looked gray and by shifting to the center became yellow, then greenish and then completely green.

Blue and yellow are seen on parts that are remote from the center point compared to red and green, which means that blue and yellow are more visible than red and green on the outer periphery edges [6]. 
Such result was expected with regarding the architecture of the eye. There are two types of photoreceptors - cone and rod cells. Near the fovea the ratio of the rods and the cones is $2: 1$, while the periphery decreases the number of cones so the lateral parts of the retina dominate the rods which react to the dim light, characterized by a lower resolution of the vision, difference between black and white as well as the night vision.

\section{THEORETICAL PART}

\subsection{Color}

Color has a key role in digital visual information and communication, so it is important to know the numerous color characteristics. Color has long been studied in a number of disciplines, but in the field of computer vision a lot shorter. By development of technology in digital cameras, printers and other devices, black and white screens are rarely used and the use of color in computer vision becomes daily [7].

The same shade of color does not manifest itself equally in all parts of the screen, but the human eye is not sufficiently sensitive to the gradual changes in color shades. For that reason, experiments are sometimes performed so that the constant color value is displayed on different parts of the screen [8].

\subsection{Peripheral Vision}

The peripheral vision area is very important for observation and reading, although the greatest visual acuity is in the fovea.

Visual acuity, resolution, and color vision are weaker in the peripheral space, respectively decrease bydistance from the point of fixation [9]. Everything that is visible to the eye outside the fovea when the eye is focused on a certain point is located in the peripheral vision area [6] "Foveal vision subtends a very small angle of view (Fig. 1), while impressions in the parafovea are somewhat less distinct. Stimuli in peripheral vision are poorly resolved, but movement of objects in the periphery significantly enhances our ability to detect them." [10]

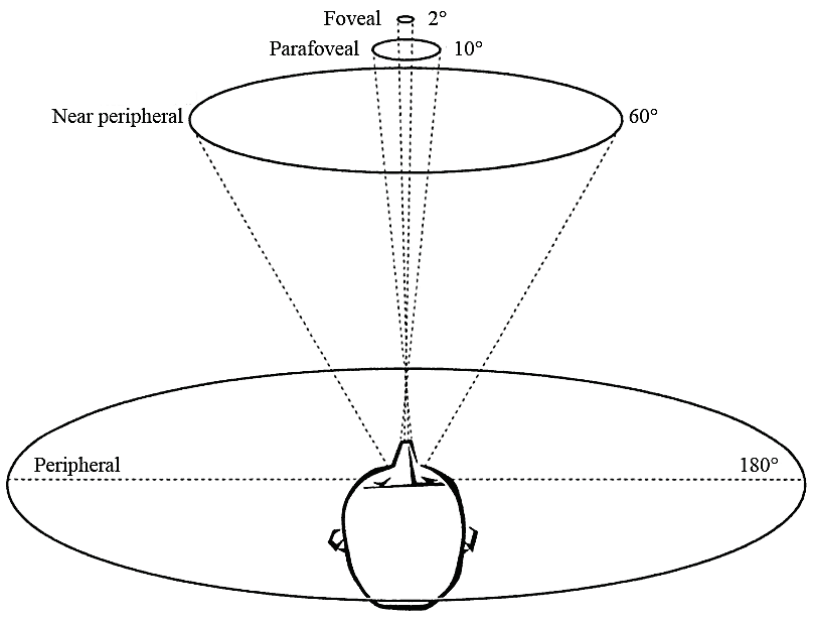

Figure 1 Demonstration of foveal, parafoveal, near peripheral and peripheral vision [10]

The human eye with normal vision has a higher resolution in fovea than some digital cameras, and can discern several thousand points within that area. Outside the fovea, at the distance of length of the extended arm, the resolution is only a few tenth points per inch [11].

\section{EXPERIMENT}

The purpose of this experiment was to examine the influence of color and shape on the identification of moving objects in the peripheral part of the screen. The attention of the examinees was focused on the central part of the screen, and in the area of peripheral vision there were randomly displayed elements which influenced the attention of the examinees by their appearance and motion.

Testing was conducted at daylight in a classroom with a large number of computers. The test was performed on devices that have a resolution greater than the minimum $1024 \times 768$ px (screen size $21 "$ to $24 "$ at an average distance of $60 \mathrm{~cm}$ from the screen).

For the purpose of the research, a web application has been developed, consisting of a graphical interface and algorithm (set of rules) developed in the ActionScript 3.0 program language, which allows to load XML (Extensible Markup Language) forms for the purpose of creating and parameterizing the tests. On the server there is an API (Application Programming Interface), developed in the PHP (Hypertext Preprocessor) language, for storing data in the MySQL (open source relational database management system) database. That way, respondents were able to access the test using the unique URL (Uniform Resource Locator) link and all the data was stored in one place.

Through the XML forms, for each individual test, it is possible to determine the range (min and max) of each parameter in which the application randomly selected the values for each examinee. That way, each examinee received a differently parameterized test during experiment.

The test was designed so that it was necessary to fill in user information at the beginning and short instructions were provided before initiating the first step.

When an examinee was ready, they independently started each test. Immediately before the start of the test, a five-second countdown was scheduled for the preparation of the examinee. After three tests were completed, there was a part where examinee could provide feedback regarding duration and comprehensibility of the testing. The data generated in this way provided the guidelines for further research. Moreover, at the very end of the experiment, examinees could see the results of their testing.

174 students of UniversityNorth in Varaždin did the test, out of which 132 were male and 42 female of an average age of 22 .

\subsection{The Design and Characteristics of Tests}

In this chapter, we will briefly present the design and characteristics of each of the tests we used in our research and comment on the issues we encountered during the experiment.

The experiment consisted of three consecutive tests, each lasting 20 to 30 seconds and was defined by 7 parameters ranging from the minimum and maximum values (Tab. 1) 
Table 1 Parameters that affect the measurement of the resolution in the test (abbreviations for stimulus movement direction are presented in Tab. 2)

\begin{tabular}{|c|c|}
\hline Parameter & Min and max value \\
\hline Time of appearance of stimulus & $20-30 \mathrm{sec}$ from start \\
\hline $\begin{array}{c}\text { The time needed for the stimulus to } \\
\text { pass the path }\end{array}$ & $2-4 \mathrm{sec}$ \\
\hline The size of the stimulus in pixels & $80-100 \mathrm{px}$ \\
\hline $\begin{array}{c}\text { Moving direction of the stimulus } \\
\text { (Tab. 2) }\end{array}$ & $\begin{array}{c}\mathrm{DL} / \mathrm{UL}, \mathrm{UL} / \mathrm{DL}, \mathrm{UL} / \mathrm{UR}, \\
\mathrm{UR} / \mathrm{UL}, \mathrm{UR} / \mathrm{DR}, \mathrm{DR} / \mathrm{UR}, \\
\mathrm{DL} / \mathrm{DR}, \mathrm{DR} / \mathrm{DL}\end{array}$ \\
\hline Rotation of the stimulus & $0^{\circ}-45^{\circ}$ \\
\hline Color of the stimulus & black, yellow, red, green, blue \\
\hline Shape of the stimulus & $\begin{array}{c}\text { triangle, square, hexagon, } \\
\text { octagon }\end{array}$ \\
\hline
\end{tabular}

Table 2 Movement directions of the stimulus

\begin{tabular}{|c|c|c|c|}
\hline $\begin{array}{c}\text { Movement direction } \\
\text { of the stimulus }\end{array}$ & $\begin{array}{c}\text { Abbreviati } \\
\text { on of } \\
\text { direction }\end{array}$ & $\begin{array}{c}\text { Movement direction of } \\
\text { the stimulus }\end{array}$ & $\begin{array}{c}\text { Abbreviati } \\
\text { on of } \\
\text { direction }\end{array}$ \\
\hline down left-up left & DL/UL & up right - down right & UR/DR \\
\hline up left - down left & UL/DL & down right- up right & DR/UR \\
\hline up left - up right & UL/UR & down left - down right & DL/DR \\
\hline up right - up left & UR/UL & down right - down left & DR/DL \\
\hline
\end{tabular}

All tests were performed so that a particular task is displayed black text on a white background. This color combination was chosen because previous studies $[12,13]$ show that the combination of black text on a white background was confirmed as being one of the best for reading. In these experiments, examinees had the smallest number of reading mistakes in case of black text on the white background, and it was confirmed that this color combination was least fatiguing for the eyes.

The tests we used were designed so that a particular task, which the examinee needs to solve, is displayed in the center of the screen, thus focusing the attention on that part of the screen. Namely, the idea is to narrow the examinees' attention to the central part of the screen by actively solving a simple task. The examinee has been previously instructed to terminate the test at a moment when he or she observes an object moving from one part of the screen to another. After the completion of the tests, it was obligatory to answer five questions:

1) How many differences are there in the pictures?

2) How many triangles are there in the picture?

3) How many mistyped words are there in the text?

4) What kind of shape was noticed in the peripheral part?

5) What was the color of the shape?

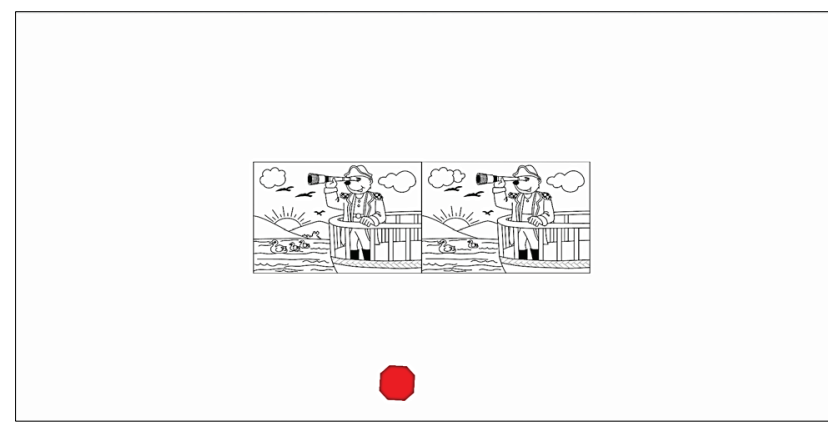

Figure 2 Example of the first test

There were three different examples for each test, which appeared randomly, ensuring that the same example did not appear to all examinees.

The task of the first test was to count how many differences there are in seemingly two equal pictures. It was possible to find ten differences in each of the three examples (Fig. 2).

The task of the second test was to count the triangles in the picture. The task was more complex than it looked, because it actually contained a larger number of triangles than it seemed at first glance. In this test, each example had a different number of responses (depending on the example, the exact number of triangles was 10,13 or $16-$ Fig. 3).

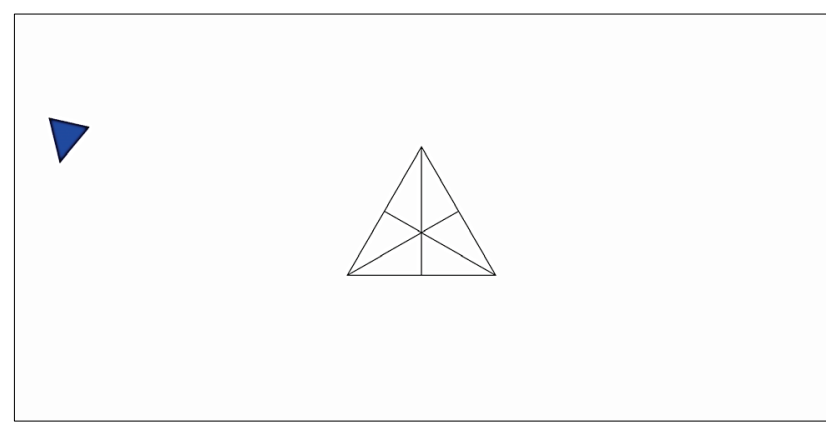

Figure 3 Example of the second test

The task of the third test was to count the words which are mistyped in the text. Each of the three examples had seven mistyped words in the text. The text is written in Arial black on a white background (Fig. 4). This font has been used since it was proven that sans serif fonts (Arial and Verdana) were shown to be easier to read on digital devices (tablets and personal computers) $[14,15]$.

This test has encountered problem with detecting spelling mistakes. Namely, it was possible to find seven spelling mistakes, but $19,5 \%$ of examinees found more than seven mistakes or found no mistakes. Because of the nature of the task, it was better to avoid such a type of test because some examinees have an obvious problem in finding the spelling mistakes.

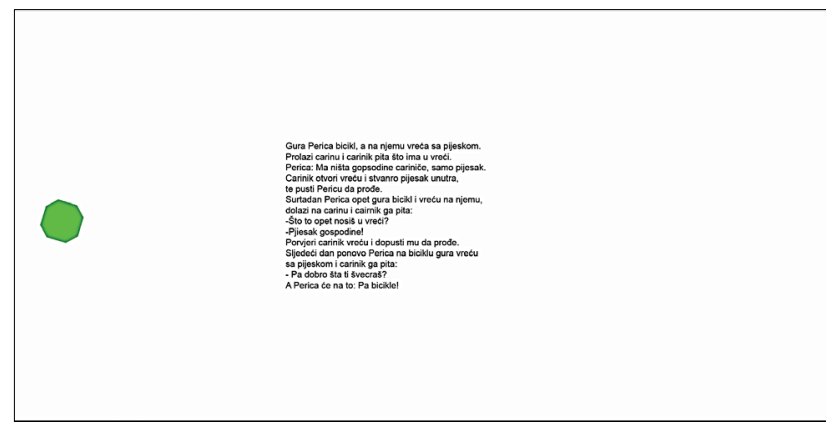

Figure 4 Example of the third test

\subsection{Stimulus in Peripheral Field}

To test the perception quality of objects in the peripheral part of the screen, on the previously described tests, we superimposed objects (different in color, shape and speed) that were moving along the edge of the screen.

In order to achieve excellent peripheral readability, and for the purpose of searching the information contained in the peripheral space, motion elements have been designed $[16,17,18]$.

For this reason, in these measurements, the stimulus in the peripheral part of the screen was movable. 
In each test, the stimulus was triggered in one of eight randomly selected directions on the peripheral part of the screen (Tab. 2).

In addition to the different motion directions, the stimuli in the peripheral part of the screen appeared randomly in five different colors (black, yellow, red, green and blue) and four different shapes (triangle, square, hexagonal and octagonal). After the tests were completed, examinees offered answers in the drop-down menu to determine the color of the shape and types of stimuli they saw, after which the experiment for each subject was completed. There were five colors and six different shapes offered as responses. Pentagon and heptagon were also included in the set, even though they did not appear in these tests. Reason for that is to test how much the examinees are confident in their answer.

\section{RESULTS}

The first step in our experiment was to examine the accuracy of determining the shape of an individual stimulus (Fig. 5). The highest percentage of correctly estimated stimulus had a triangle $(93,96 \%)$ and the lowest had an octagon $(34,88 \%)$, which points to the fact that complex forms are more difficult to perceive in the peripheral space. (Tab. 3).

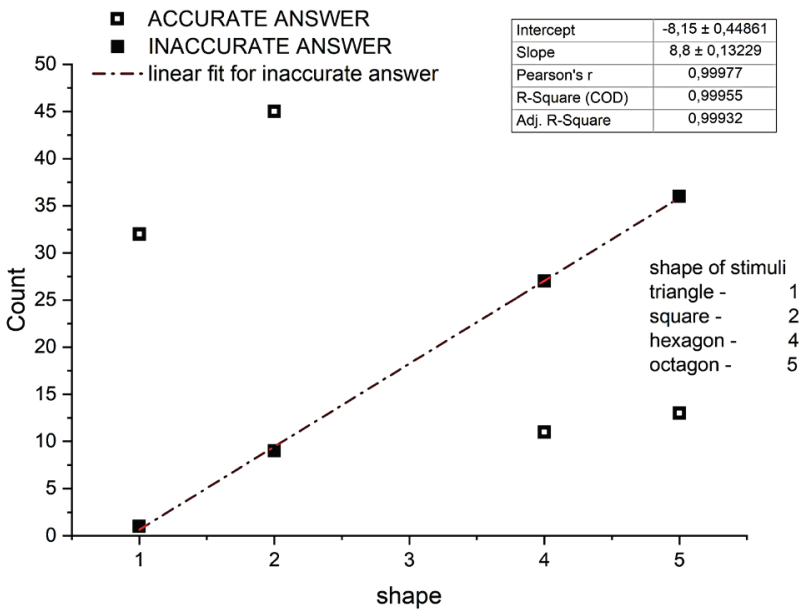

Figure 5 Display of correctly perceived shapes

The fact that polygons with more angles were harder to distinguish is also confirmed by the fact that in as many as $10 \%$ of the cases, the pentagon was chosen as a response in the case of hexagons or octagons. Heptagon was selected only in $1,5 \%$ of cases. It is interesting to note that the number of incorrect answers increases linearly with the number of polygonal sides, indicating that more complex forms are harder to discriminate in the peripheral space. At this instance we are unable to offer plausible explanation for this behavior and we intend to explore this behavior in one of the following experiments.

Table 3 Shape of stimulus in peripheral field

\begin{tabular}{|c|c|c|c|c|}
\hline $\begin{array}{c}\text { Shape of } \\
\text { stimulus in } \\
\text { peripheral field }\end{array}$ & $\begin{array}{c}\text { Average } \\
\text { value (\%) }\end{array}$ & $\begin{array}{c}\text { Accurate } \\
\text { shape in } \\
\text { test 1 (\%) }\end{array}$ & $\begin{array}{c}\text { Accurate } \\
\text { shape in } \\
\text { test 2 (\%) }\end{array}$ & $\begin{array}{c}\text { Accurate } \\
\text { shape in } \\
\text { test 3 (\%) }\end{array}$ \\
\hline Triangle & 93,96 & 96,97 & 100 & 84,91 \\
\hline Square & 77,98 & 83,33 & 67,27 & 83,33 \\
\hline Hexagon & 42,64 & 28,95 & 57,5 & 41,46 \\
\hline Octagon & 34,88 & 26,53 & 43,9 & 34,21 \\
\hline
\end{tabular}

Analysis of the results of the perceived color perception of stimuli in our tests did not show significant deviations and all colors had a high percentage of correct answers. (Tab. 4)

One of the earliest papers shows that when a red stimulus appears in the peripheral part, the users identify the color much more easily [5].

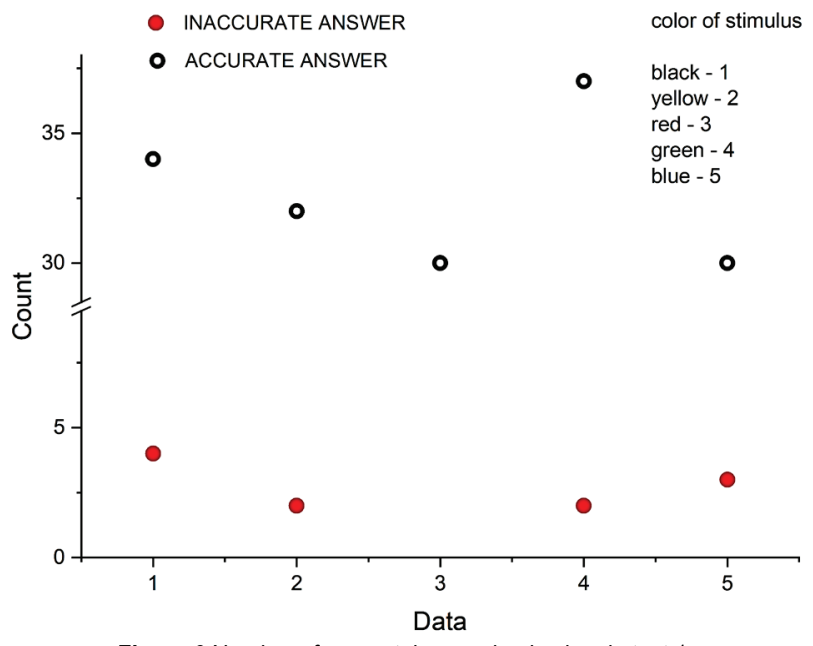

Figure 6 Number of accurately perceived colors in test 1 .

Table 4 Color of stimulus in peripheral field

\begin{tabular}{|c|c|c|c|c|}
\hline $\begin{array}{c}\text { Color of } \\
\text { stimulus in } \\
\text { peripheral field }\end{array}$ & $\begin{array}{c}\text { Average } \\
\text { value (\%) }\end{array}$ & $\begin{array}{c}\text { Accurate } \\
\text { color in } \\
\text { test 1 (\%) }\end{array}$ & $\begin{array}{c}\text { Accurate } \\
\text { color in } \\
\text { test 2 (\%) }\end{array}$ & $\begin{array}{c}\text { Accurate } \\
\text { color in } \\
\text { test 3 (\%) }\end{array}$ \\
\hline Black & 93,41 & 89,47 & 93,99 & 96,77 \\
\hline Yellow & 93,38 & 94,12 & 92,68 & 93,33 \\
\hline Green & 93,16 & 94,87 & 92,11 & 92,5 \\
\hline Red & 92,29 & 100 & 93,10 & 83,78 \\
\hline Blue & 90,49 & 90,91 & 88,89 & 91,67 \\
\hline
\end{tabular}

The idea of this paper is to present a new approach to the research of perception of stimuli in the peripheral part of the screen, i.e. when examinee consciously consumes some content. Therefore, it was necessary to conduct research independent on the predetermined number of correct responses and the reaction time.

On the other hand, we have assumed that the results delivered by respondents whose concentration was more focused on the task (which were placed in the center of the screen) had a greater statistical weight.

A greater number of correct answers is certainly the result of a higher concentration of respondents and time that is long enough for finding mistakes in text. In this chapter we will show in detail how we made our testing independent of the above-mentioned problems.

As already mentioned, the time for doing the test was randomly assigned to the examinees. Stimuli occurred after the expiration of that time and examinees reaction time to its occurrence was measured. The duration of the individual test was 20 to 30 seconds, and the subjects whose tests lasted shorter were in a disadvantageous position than those who had a longer duration of the test. It is therefore expected that examinees who had more time to do the test would achieve a better result. Due to the need to objectively test the responses, a standardized response number was introduced. This was achieved by pondering the response with the corresponding pondering factor $\mathrm{w}$ : 
$w_{p, i}=\left(\frac{t_{\max }}{t_{\text {test }}}\right)_{p, i} \quad \begin{aligned} p & =\text { test number } \\ & =\text { respondent }\end{aligned}$

where $t_{\max }$ is the maximum time which passed before stimulus appeared and $t_{\text {test }_{i}}$ is actual time which passed before stimulus appeared. This procedure significantly reduces the impact of the length of time intended for completion of the test (by random selection, some respondents had more and some less time to complete the test). The next correction is reflected in the additional weighting of responses. The next problem was to determine what the concentration level of the examinees was during the completion of the particular test. This was achieved by "honoring" the examinees with more accurate answers. The assumption is that the examinee with greater focus (concentration) on the center of the screen (problem) will achieve better results in doing the test. The pondering factor $w_{\text {correct }_{p, i}}$ thus obtained had a form:

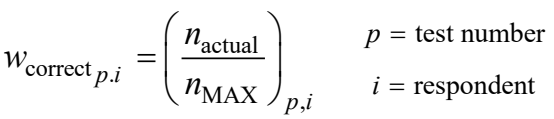

$n_{\text {actual }}$ is the number of responses recorded by an individual examinee, and $n_{\mathrm{MAX}}$ is the maximum number of correct answers. Thus obtained weight factors gave a single weight factor $W_{p, i}$ formed:

$W_{p, i}=w_{p, i} \cdot w_{\text {correct }}^{p, i} \quad i=$ respodent

The pondering factor $W$ thus defined balances the influence of the concentration of the examinees and the length of the time received to do the test for every examinee. Finally, by multiplying the number of responses of each individual respondent with pondering factor $W$, we get the final socalled normalized response number for each examinee.

$N_{\text {norm }}=n_{\text {actual }} \cdot W$

or

normalized number of responses $=$ col $^{\prime \prime}$ the answer to the question in the first test $" \times$

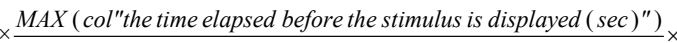
col"the time elapsed before the stimulus is displayed ( $\left.(\mathrm{sec})^{\prime \prime}\right)$ col "the answer to the question in the first test"

$\times$ MAX (col "the answer to the question in the first test")

\section{DISCUSSION}

The result of this pondering approach is best seen by comparing non-normalized and normalized responses as reaction time functions in test 1 (Fig. 7 and Fig. 8).

It is obvious that the presented method largely eliminates the problem of a predetermined number of correct answers in a particular test and can be generalized for an arbitrary number of correct answers. Otherwise, it would be necessary to argument why the test is designed so that it has exactly eight correct answers, or whether it is the optimal number of correct answers or it should be another number. In order to avoid this, we have presented the results in percentages of accurately weighted responses (Fig. 9).

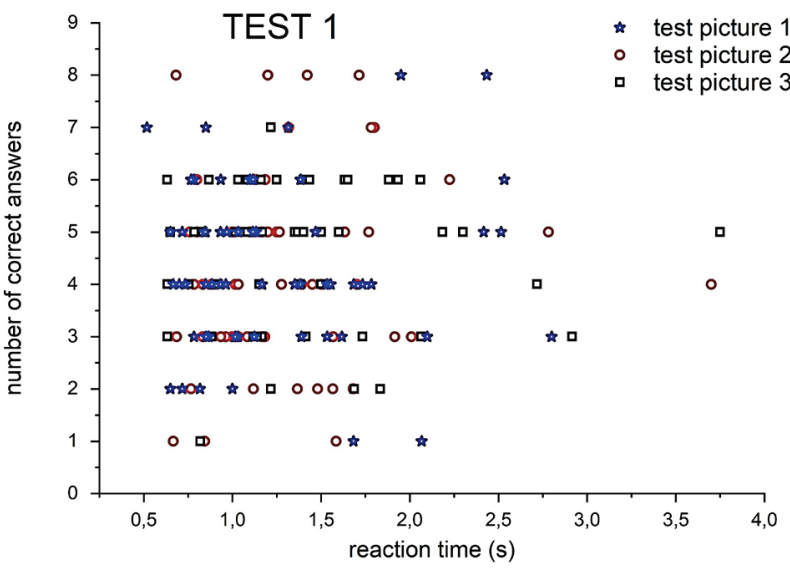

Figure 7 Number of correct answers as reaction time function for all three images in test 1

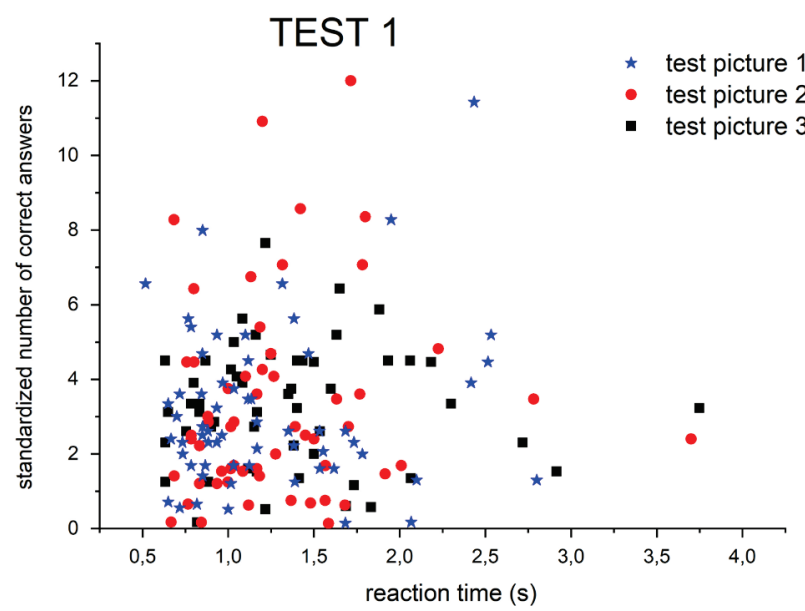

Figure 8 Number of normalized correct responses as reaction time function for all three images in test 1

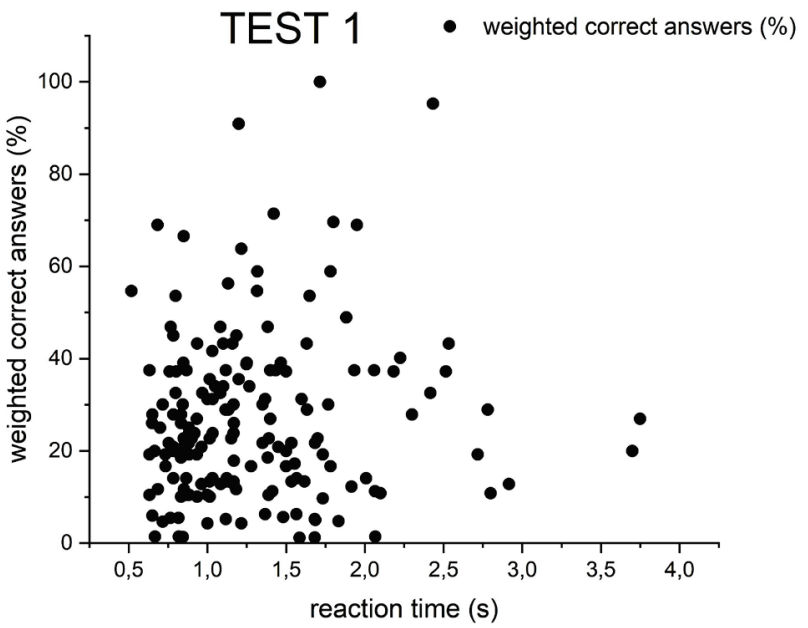

Figure 9 Percentage of standardized correct answers as reaction time function for all images in test 1

Statistical analysis yielded the mean value and standard deviation of the percentage of weighted correct answers for Test 1:

$\operatorname{corr}_{\mathrm{ans}}=(26,73 \pm 18,16) \%$

which were obtained with the reaction time: 
time $_{\text {reaction }}(\mathrm{s})=(1,29 \pm 0,56) \mathrm{s}$

The results obtained for the percentage distribution of standardized correct answers (for Test 1) are shown in Fig. 10. Similar analysis was also performed for the corresponding reaction time.

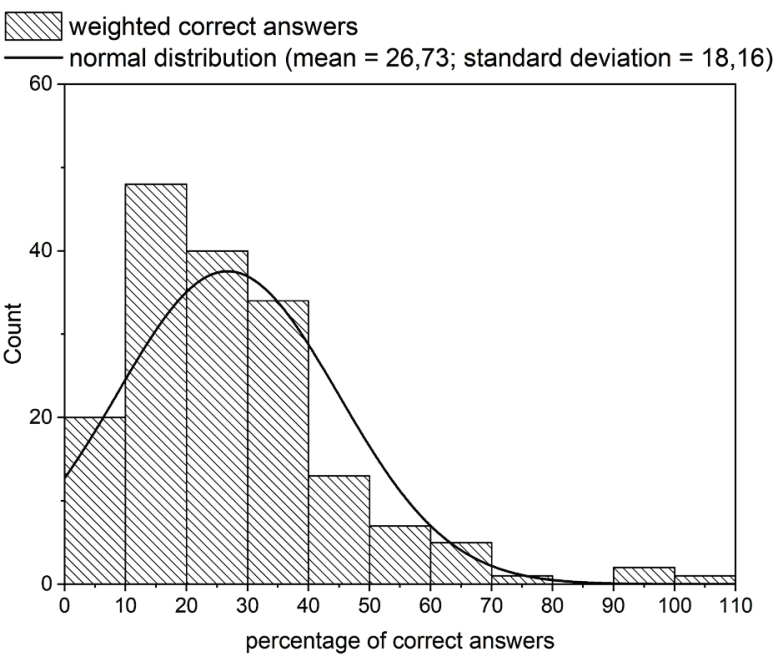

Figure 10 Standardized correct answers percentage distribution fit corresponding to normal distribution and histogram of standardized correct responses percentage in test 1

Figs. 9 and 10 clearly show that the reaction time for Test 1 falls in the interval between 0,7 and 1,9 seconds. This cannot be determined from Fig. 7 which shows nonnormalized responses number as a function of examinee reaction time to stimuli appearance. Furthermore, Fig. 8 shows that the reaction time does not depend on the offered test image. However, the origin and cause of the surprisingly small percentage of correct answers will be the subject of further research into the dependence of weighted responses on the type of test.

\section{CONCLUSION}

The paper clearly shows that the method of introducing standardization of the correct answers, in the test, by pondering certain factors that affects the final test result, generates clear and generally applicable results. The design of our test required that the presented method must be applied to the number of correct answers and the time the respondent had available to do the test. One pondering procedure responded to a greater number of correct responses as a measure of the concentration of respondents on the task, and the second put the respondents in equal position regarding the consumption of time to complete the test.

In several experiments $[4,19,20]$ only one point in the center of the screen was used to ensure that the respondents focus their attention to that place. However, in a recent work [21], examinees should be focused, for the same purpose, between two centered points in the center of the screen (distance between two points: $3^{\circ}$ ).

Such an approach, in our opinion, is not efficient enough and does not yield plausible results. Focusing the attention of the respondent on the central dot does not require any mental activity, and no one can say for sure that the respondent does not "expect" some kind of action. But in real situations this is not the case. We are always, more or less, focused on some content on the screen. If content is more interesting or challenging, our attention is more focused on the part of the screen where this content is. We have achieved this by subjecting examinees to the tests that require solving different tasks in order to engage their attention.

The introduced method of the pondering factors association provides a more objective approach to the treatment of the number of correct answers in tests that are studying the reaction to perception with peripheral sight and can be considered a new approach to this problem. The number of correct answers in the test was taken as a measure of examinees' concentration on the given problem.

The idea of the paper was to examine to what extent we can use perception with peripheral sight in, for example, commercial purposes, while keeping our attention to the preferred content on the screen. As this was a preliminary measurement, the plan for further research is to conduct a more detailed analysis of how font changes in the peripheral part of the screen affect the readability and design of the user interface. The focus of the research will be on the analysis and parameterization of different font sizes, paragraph sizes, kerning, tracking and text colors.

This and further researches will provide useful guidelines for web and digital content as designers will get new opportunities in designing less visible parts of the screen. Peripheral space can have great application in creating quality content, for example, in the area of key information and advertising in digital environment. The Internet is a great place to advertise, but large-format banners often hinder the attention of the user. Proper use of colors, shapes and fonts ensures functionality of the peripheral part without hindering the user whose focus is on the central part of the screen.

Researchers have found a significant improvement in peripheral awareness of people who played computer games specially designed around using peripheral vision. Therefore, this research can also make a significant contribution to the computer games industry.

\section{REFERENCES}

[1] Pavlovskaya, M., Ring, H., Groswasser, Z., Keren, O. \& Hochstein, S. (2001). Visual search in peripheral vision: Learning effects and set-size dependence. Spatial Vision, 14(2), 151-173. https://doi.org/10.1163/156856801300202913

[2] Gutwin, C., Cockburn, A., \& Coveney, A. (2017). Peripheral popout: The influence of visual angle and stimulus intensity on popout effects. Proceedings of the 2017 CHI Conference on Human Factors in Computing Systems (pp. 208-219). ACM. https://doi.org/10.1145/3025453.3025984

[3] Levi, D. M., Klein, S. A., \& Aitsebaomo, A. P. (1985). Vernier acuity, crowding and cortical magnification. Vision research, 25(7), 963-977. https://doi.org/10.1016/0042-6989(85)90207-X

[4] Hansen, T., Pracejus, L., \& Gegenfurtner, K. R. (2009). Color perception in the intermediate periphery of the visual field. Journal of Vision, 9(4), 26, 1-12. https://doi.org/10.1167/9.4.26

[5] Gordon, J. \& Abramov, I. (1977). Color vision in the peripheral retina. II. Hue and saturation. JOSA, 67(2), 202207. https://doi.org/10.1364/JOSA.67.000202 
[6] Frey, N. W. (1932). A comparative study of the color zones and the form zones in peripheral vision. Doctoral dissertation, Massachusetts State College.

[7] Gevers, T., Gijsenij, A., Van de Weijer, J., \& Geusebroek, J. M. (2012). Color in computer vision: fundamentals and applications (Vol. 23). John Wiley \& Sons. https://doi.org/10.1002/9781118350089

[8] Hunt, R. W. G. \& Pointer, M. R. (2011). Measuring Color ( $4^{\text {th }}$ Edition), John Wiley \& Sons. https://doi.org/10.1002/9781119975595

[9] Rosenholtz, R. (2016). Capabilities and limitations of peripheral vision. Annual Review of Vision Science, 2, 437457. https://www.annualreviews.org/doi/10.1146/annurev-vision082114-035733

[10] Solso, R. L. (1996). Cognition and the visual arts. The MIT press. Cambridge, London

[11] Johnson, J. (2010). Designing with the mind in mind: simple guide to understanding user interface design rules. Elsevier. https://doi.org/10.1016/C2009-0-20318-7

[12] Zjakić, I. \& Milković, M. (2010). Psihologijaboja, Veleučilište u Varaždinu, ISBN 978-953-95000-1-4

[13] Zorko, A., Ivančić Valenko, S., Tomiša, M., \& Keček, D., Cerepinko, D. (2017). The impact of the text and background color on the screen reading experience. Technical Journal, 11(3), 78-82.

[14] Čerepinko, D., Periša, M., \& Keček, D. (2017). Text readability and legibility on iPad with comparison to paper and computer screen. Tehnički vjesnik, 24(4), 1197-1201. https://doi.org/10.17559/TV-20160225140202

[15] Sheedy, J. E., Subbaram, M. V., Zimmerman, A. B., \& Hayes, J. R. (2005). Text legibility and the letter superiority effect. Human Factors: The Journal of the Human Factors and Ergonomics Society, 47(4), 797-815. https://doi.org/10.1518/001872005775570998

[16] Alwitt, L. F. (1976). Selective attention in the peripheral visual field. Doctoral dissertation, University of Massachusetts Amherst.

[17] Finlay, D. (1982). Motion perception in the peripheral visual field. Perception, 11(4), 457-462. https://doi.org/10.1068/p110457

[18] Kooi, F. L. \& Mosch, M. (2006, October). Peripheral motion displays: tapping the potential of the visual periphery. Proceedings of the Human Factors and Ergonomics Society Annual Meeting (Vol. 50, No. 16, pp. 1604-1608). Sage CA: Los Angeles, CA: SAGE Publications. https://doi.org/10.1177/154193120605001619

[19] Anstis, S. M. (1974). A chart demonstrating variations in acuity with retinal position. Vision research, 14(7), 589-592. https://doi.org/10.1016/0042-6989(74)90049-2

[20] Bernard, J. B., Aguilar, C., \& Castet, E. (2016). A new font, specifically designed for peripheral vision, improves peripheral letter and Word recognition, but not eye-mediated reading performance. PloS one, 11(4), e 0152506. https://doi.org/10.1371/journal.pone.0152506

[21] Bernard, J. B. \& Castet, E. (2019). The optimal use of nonoptimal letter information in foveal and parafoveal word recognition. Vision research, 155, 44-61. https://doi.org/10.1016/j.visres.2018.12.006

\section{Contact information:}

Snježana IVANČIĆ VALENKO, dipl. ing., lecturer Corresponding author

University North,

Trg dr. Žarka Dolinara 1, 48000 Koprivnica, Croatia

snjezana.ivancic@unin.hr

Vladimir CVILJUŠAC, mag. ing. techn. graph University of Zagreb, Faculty of Graphics Arts, Getaldićeva 2, 10000 Zagreb, Croatia

vladimir.cviljusac@grf.hr

Sanja ZLATIĆ, dipl. ing., lecturer

University North,

Trg dr. Žarka Dolinara 1, 48000 Koprivnica, Croatia

sanja.zlatic@unin.hr

Damir MODRIĆ, asocc. prof. dr. sc.

University of Zagreb, Faculty of Graphics Arts,

Getaldićeva 2, 10000 Zagreb, Croatia

damir.modric@grf.hr 